\title{
Association of Body Weight Variability with Adverse Cardiovascular Outcomes in Patients with Pre-Dialysis Chronic Kidney Disease
}

\author{
Sang Heon Suh ${ }^{1} \mathbb{D}$, Tae Ryom Oh ${ }^{1}$, Hong Sang Choi ${ }^{1} \mathbb{D}$, Chang Seong Kim ${ }^{1}{ }^{\mathbb{D}}$, Eun Hui Bae ${ }^{1}$, Sue K. Park ${ }^{2,3,4} \mathbb{D}$, \\ Yong-Soo Kim ${ }^{5}{ }^{(0)}$, Yeong Hoon Kim ${ }^{6}$, Kyu Hun Choi ${ }^{7}$, Kook-Hwan $\mathrm{Oh}^{8}{ }^{\circledR}$, Seong Kwon Ma ${ }^{1, *}$, \\ Soo Wan Kim ${ }^{1, *}$ and on behalf of the KoreaN Cohort Study for Outcomes in Patients With Chronic Kidney \\ Disease (KNOW-CKD) Investigators ${ }^{\dagger}$
}

check for updates

Citation: Suh, S.H.; Oh, T.R.; Choi, H.S.; Kim, C.S.; Bae, E.H.; Park, S.K.; Kim, Y.-S.; Kim, Y.H.; Choi, K.H.; Oh, K.-H.; et al. Association of Body Weight Variability with Adverse Cardiovascular Outcomes in Patients with Pre-Dialysis Chronic Kidney Disease. Nutrients 2021, 13, 3381. https://doi.org/10.3390/nu13103381

Academic Editor: Manuela Abbate

Received: 6 September 2021

Accepted: 20 September 2021

Published: 26 September 2021

Publisher's Note: MDPI stays neutral with regard to jurisdictional claims in published maps and institutional affiliations.

Copyright: (C) 2021 by the authors. Licensee MDPI, Basel, Switzerland. This article is an open access article distributed under the terms and conditions of the Creative Commons Attribution (CC BY) license (https:/ / creativecommons.org/licenses/by/ $4.0 /)$.
1 Department of Internal Medicine, Chonnam National University Medical School and Chonnam National University Hospital, Gwangju 61469, Korea; medssh1984@gmail.com (S.H.S.); tryeomoh@hanmail.net (T.R.O.); hongsang38@hanmail.net (H.S.C.); laminion@hanmail.net (C.S.K.); baedak76@gmail.com (E.H.B.)

2 Department of Preventive Medicine, Seoul National University College of Medicine, Seoul 03080, Korea; suepark@snu.ac.kr

3 Cancer Research Institute, Seoul National University, Seoul 03080, Korea

4 Integrated Major in Innovative Medical Science, Seoul National University College of Medicine, Seoul 03080, Korea

5 Department of Internal Medicine, College of Medicine, Catholic University of Korea, Seoul 06591, Korea; kimcmc@catholic.ac.kr

6 Department of Internal Medicine, Busan Paik Hospital, College of Medicine, Inje University, Busan 47392, Korea; yeonghnl@inje.ac.kr

7 Department of Internal Medicine, College of Medicine, Institute of Kidney Disease Research, Yonsei University, Seoul 03722, Korea; Khchoi6@yuhs.ac

8 Department of Internal Medicine, Seoul National University Hospital, Seoul 03080, Korea; ohchris@hanmail.net

* Correspondence: drmsk@hanmail.net (S.K.M.); skimw@chonnam.ac.kr (S.W.K.); Tel.: +82-62-220-6579 (S.K.M.); +82-62-225-6271 (S.W.K.)

+ The KoreaN Cohort Study for Outcomes in Patients With Chronic Kidney Disease (KNOW-CKD) Investigators are listed in Acknowledgments.

Abstract: To investigate the association of body weight variability (BWV) with adverse cardiovascular (CV) outcomes in patient with pre-dialysis chronic kidney disease (CKD), a total of 1867 participants with pre-dialysis CKD from Korean Cohort Study for Outcomes in Patients With Chronic Kidney Disease (KNOW-CKD) were analyzed. BWV was defined as the average absolute difference between successive values. The primary outcome was a composite of non-fatal CV events and all-cause mortality. Secondary outcomes were fatal and non-fatal CV events and all-cause mortality. High BWV was associated with increased risk of the composite outcome (adjusted hazard ratio (HR) 1.745, 95\% confidence interval (CI) 1.065 to 2.847 ) as well as fatal and non-fatal CV events (adjusted HR $1.845,95 \%$ CI 1.136 to 2.996 ) and all-cause mortality (adjusted HR 1.861, 95\% CI 1.101 to 3.145). High BWV was associated with increased risk of fatal and non-fatal CV events, even in subjects without significant body weight gain or loss during follow-up periods (adjusted HR 2.755, 95\% CI 1.114 to 6.813$)$. In conclusion, high BWV is associated with adverse CV outcomes in patients with pre-dialysis CKD.

Keywords: all-cause mortality; body weight variability; cardiovascular events; chronic kidney disease

\section{Introduction}

Patients with chronic kidney disease (CKD) are likely to have experienced body weight fluctuations. Body weight (BW) loss associated with malnutrition-inflammation is 
prevalent even before the commencement of renal replacement therapy [1,2] and increases mortality [3]. Several factors are associated with appetite impairment in patients with CKD, which further contributes to protein-energy wasting [4]. Conversely, BW gain associated with excess extracellular fluid is also common in patients with CKD, resulting in accelerated coronary artery calcification [5] and increased all-cause mortality [6]. The prevalent use of diuretics in CKD patients further impose the likelihood of body weight variability (BWV) [7]. As these conditions are not mutually exclusive, it could be assumed that a considerable portion of patients with CKD may experience fluctuations in their BW during the progression of $C K D$, rather than persistent gain or loss of BW. Nevertheless, the clinical impact of BWV in patients with pre-dialysis CKD has not been established.

BWV is an emerging predictor of adverse cardiovascular (CV) outcomes in various clinical contexts, although its probable association with health outcomes has long been suggested in general population [8]. A prospective cohort study reported that BWV is associated with all-cause mortality, and, in a subgroup with body mass index (BMI) $<25 \mathrm{~kg} / \mathrm{m}^{2}$ at the baseline, is also associated with increased risks of incident diabetes mellitus (DM) $[9,10]$ and atrial fibrillation [11] in general population. A recent nationwide cohort study reported that BWV is associated with increased risks of myocardial infarction, stroke, and all-cause mortality in patients with type $2 \mathrm{DM}[12,13]$ and in patients with in non-alcoholic fatty liver disease [14]. Fluctuation in BW is associated with a higher rate of cardiovascular (CV) events independent of traditional cardiovascular risk factors in patients with coronary artery disease (CAD) [15,16]. The association between BWV and CV outcomes, however, remains to be elucidated in patients with CKD.

We here investigated the association of BWV with CV outcomes in patients with pre-dialysis CKD. As the patients with CKD are prone to experience BW gain or loss during the course of the disease, we analyzed the association between BWV and longitudinal changes of BW in patients with CKD. We also analyzed the association of BWV with CV outcomes in patients without significant BW gain or loss during follow-up periods.

\section{Materials and Methods}

\subsection{Study Designs and Data Collection from Participants}

The Korean Cohort Study for Outcomes in Patients With Chronic Kidney Disease (KNOW-CKD) is a nationwide prospective cohort study involving 9 tertiary-care general hospitals in Korea [17]. Korean patients with CKD from stage 1 to pre-dialysis stage 5, who voluntarily provided informed consent were enrolled. The study was conducted in accordance with the principles of the Declaration of Helsinki, and the study protocol was approved by the institutional review boards of participating centers. A total of 2238 subjects were longitudinally followed up. (Figure 1). After excluding those lacking the baseline measurement of BW, and those with the number of body weight measurement during follow-up periods less than three, 1867 subjects were finally included for the analyses. The median follow-up duration was 6.155 years. Demographic information was collected from all eligible participants, including age, gender, comorbid conditions, and medication history (angiotensin-converting enzyme inhibitor/angiotensin II receptor blockers (ACEi/ARBs), diuretics, total number of antihypertensive drugs). Venous samples were collected following overnight fasting, to determine hemoglobin, albumin, total cholesterol, low-density lipoprotein (LDL) cholesterol, high-density lipoprotein (HDL) cholesterol, triglyceride, fasting glucose, high-sensitivity C-reactive protein (hs-CRP), 25(OH) vitamin D and creatinine levels at the baseline. Estimated glomerular filtration rate (eGFR) was calculated by Chronic Kidney Disease Epidemiology Collaboration (CKD-EPI) equation [18]. The urine albumin-to-creatinine ratio (UACR) was measured in random, preferably second-voided, spot urine samples. The $24 \mathrm{~h}$ urine protein excretion was also determined. 


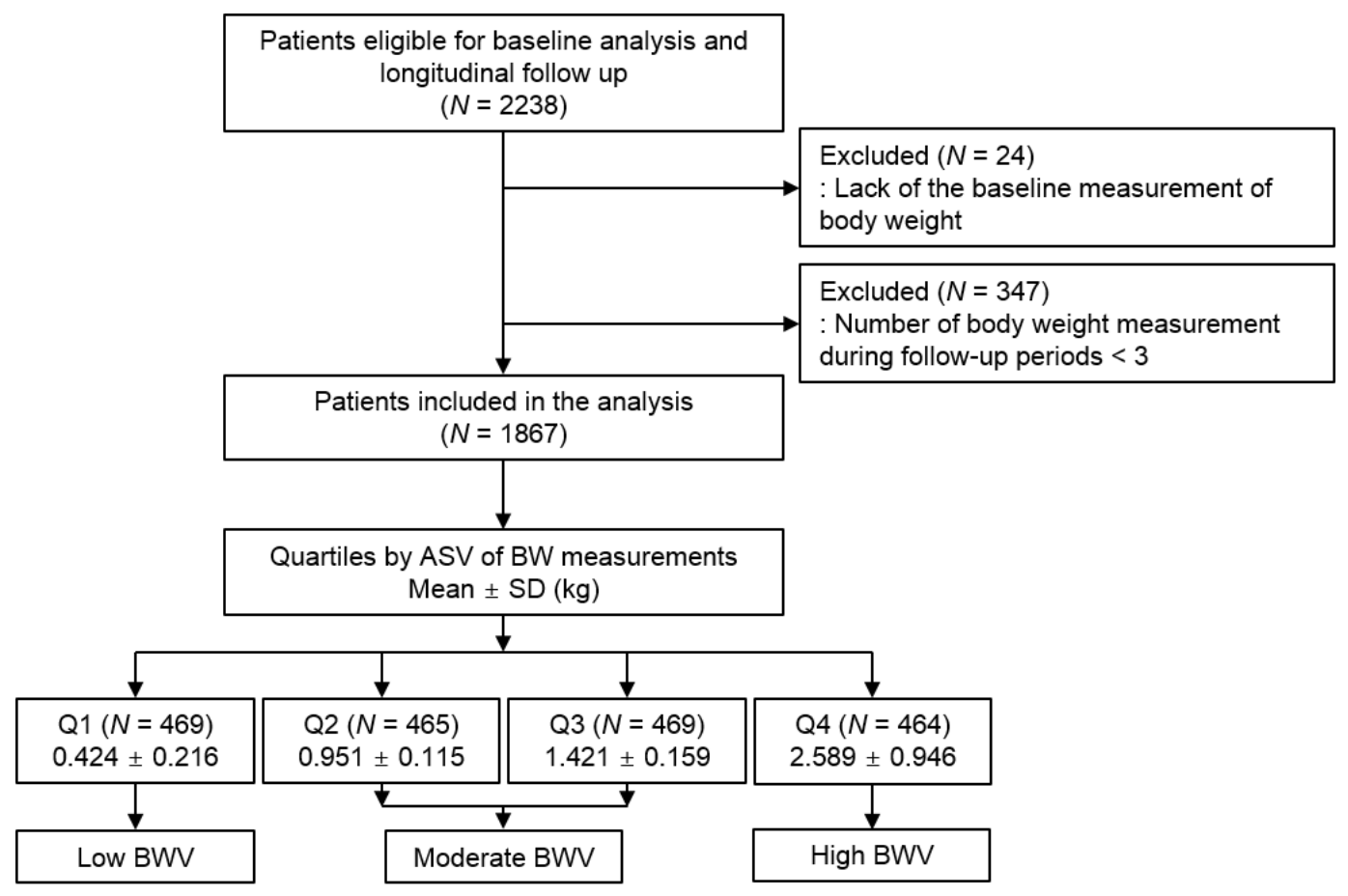

Figure 1. Flow diagram of the study participants. Abbreviations: ASV, average successive variability; BW, body weight; BWV, body weight variability; SD, standard deviation; Q1, 1st quartile; Q2, 2nd quartile; Q3, 3rd quartile; Q4, 4th quartile.

\subsection{Determination of $B W V$}

BW was measured at 0,6 , and 12 months and then yearly thereafter up to 8 years. The median number of BW measurement was 6 times. Intra-individual BWV between visits was determined by average successive variability (ASV), defined as the average absolute difference between successive values $[9,10,15,16]$. The 1st, 2 nd and 3 rd, and 4 th quartiles were defined as low, moderate, and high BWV, respectively (Figure 1).

\subsection{Estimation of the Rate of Longitudinal BW Change during Follow-Up Periods}

The rate of longitudinal BW change for each individual were estimated using a regression model and expressed as the slope (kg/year) [19]. The 1st, 2nd and 3rd, and 4 th quartiles were defined as a decrease, maintenance, and increase of BW, respectively (Supplemental Table S1).

\subsection{Study Outcomes}

The primary outcome was a composite of non-fatal CV events and all-cause mortality. Raw numbers for the composite outcome by enrollment sites subgroups are summarized in Supplemental Tables S2 and S3, respectively. Secondary outcomes were fatal and nonfatal CV events and all-cause mortality. CV events, either fatal or non-fatal, included any coronary artery event (unstable angina, myocardial infarction, or coronary intervention/surgery), hospitalization for heart failure, ischemic or hemorrhagic stroke, incident peripheral arterial disease, and symptomatic arrhythmia.

\subsection{Statistical Analysis}

Continuous variables were expressed as mean \pm standard deviation or median [interquartile range]. Categorical variables (e.g., current smoking status) were expressed as number of participants and percentage. For descriptive analyses, Student's T-test or oneway analysis of variance and $\chi^{2}$ test were used for continuous and categorical variables, respectively. The correlation between the BWV and BW slope was established by curve estimation regression analysis. Multinomial logistic regression models were analyzed to 
address the association between BWV and longitudinal BW change, where the models were adjusted for age, gender, Charlson comorbidity index, history of DM, smoking history, BMI, systolic blood pressure (SBP), diastolic blood pressure (DBP), medications (ACEi/ARBs, diuretics, total number of antihypertensive drugs), hemoglobin, albumin, HDL-cholesterol, triglycerides, fasting serum glucose, hs-CRP, 25(OH) vitamin D levels, eGFR, and $24 \mathrm{~h}$ urine protein. The results of multinomial logistic regression models were presented as odd ratios (ORs) and 95\% confidence intervals (CIs). To assess the association between BWV and the outcomes, Cox proportional hazard regression models were analyzed. Patients lost to follow-up were censored at the date of the last visit. We adjusted age, gender, Charlson comorbidity index, history of DM, smoking history, BMI, SBP, DBP, medications (ACEi/ARBs, diuretics, total number of antihypertensive drugs), hemoglobin, albumin, HDL-cholesterol, triglycerides, fasting serum glucose, hs-CRP, 25(OH) vitamin D levels, eGFR, and $24 \mathrm{~h}$ urine protein. The results of Cox proportional hazard models were presented as hazard ratios (HRs) and 95\% CIs. Statistical significance was defined as $p<0.05$. Data were analyzed using IBM SPSS statistical analysis software for Windows, version 22.0 (IBM Corp).

\section{Results}

\subsection{Baseline Characteristics}

The baseline characteristics of study participants were described by BWV (Table 1). The mean age was highest in subjects with low BWV, and lowest in subjects with high BWV. The gender distribution was not significantly different among the groups. Charlson comorbidity index was higher in subjects with high BWV. Detailed presentation of Charlson Comorbidity Index components by BWV is summarized in Supplement Table S4, which revealed that the frequency of cerebrovascular disease and diabetes with organ damage was significantly higher in the subjects with high BWV. The frequency of the subjects with previous medical history of DM was highest in the subjects with high BWV, and lowest in the subjects with low BWV. The frequencies of other medical history, such as CAD and arrhythmia were not significantly different among the groups. Smoking status did not differ across the groups either. BMI, waist circumference, and SBP were highest in the subjects with high BWV, and lowest in the subjects with low BWV. The subjects with high BWV were significantly likely to take no less than 3 antihypertensive drugs at the baseline. Serum albumin level was highest in the subjects with moderate BWV. Serum triglyceride and fasting glucose levels were highest in the subjects with high $\mathrm{BWV}$, and lowest in the subjects with low BWV. Conversely, $25(\mathrm{OH})$ vitamin D level was highest in subjects with low $B W V$, and lowest in subjects with high BWV. The $24 \mathrm{~h}$ urine protein and UACR in random urine were lowest in in the subjects with moderate BWV. Hemoglobin, total cholesterol, LDL-cholesterol, HDL-cholesterol, hs-CRP levels were not significantly different among the groups. In contrast, eGFR was highest in the subjects with high BWV, and lowest in the subjects with low BWV. Accordingly, the distribution of CKD stages across the groups was significantly different. Collectively, high BWV was in large associated with unfavorable clinical features, with an exception of eGFR, which could be attributed to significantly higher BMI in subjects with high BWV and a relevant limitation of creatinine-based estimation of glomerular filtration rate.

Table 1. Baseline characteristics of study participants by BWV.

\begin{tabular}{lcccc}
\hline & \multicolumn{3}{c}{ BWV } & \\
\hline & Low & Moderate & High & $p$ Value \\
\hline Follow-up duration (year) & $5.510 \pm 2.025$ & $6.024 \pm 1.721$ & $6.024 \pm 1.785$ & $<0.001$ \\
Age (year) & $54.889 \pm 10.969$ & $54.035 \pm 11.603$ & $51.442 \pm 13.726$ & $<0.001$ \\
Male & $278(59.3)$ & $556(59.5)$ & $299(64.4)$ & 0.161 \\
\hline
\end{tabular}


Table 1. Cont

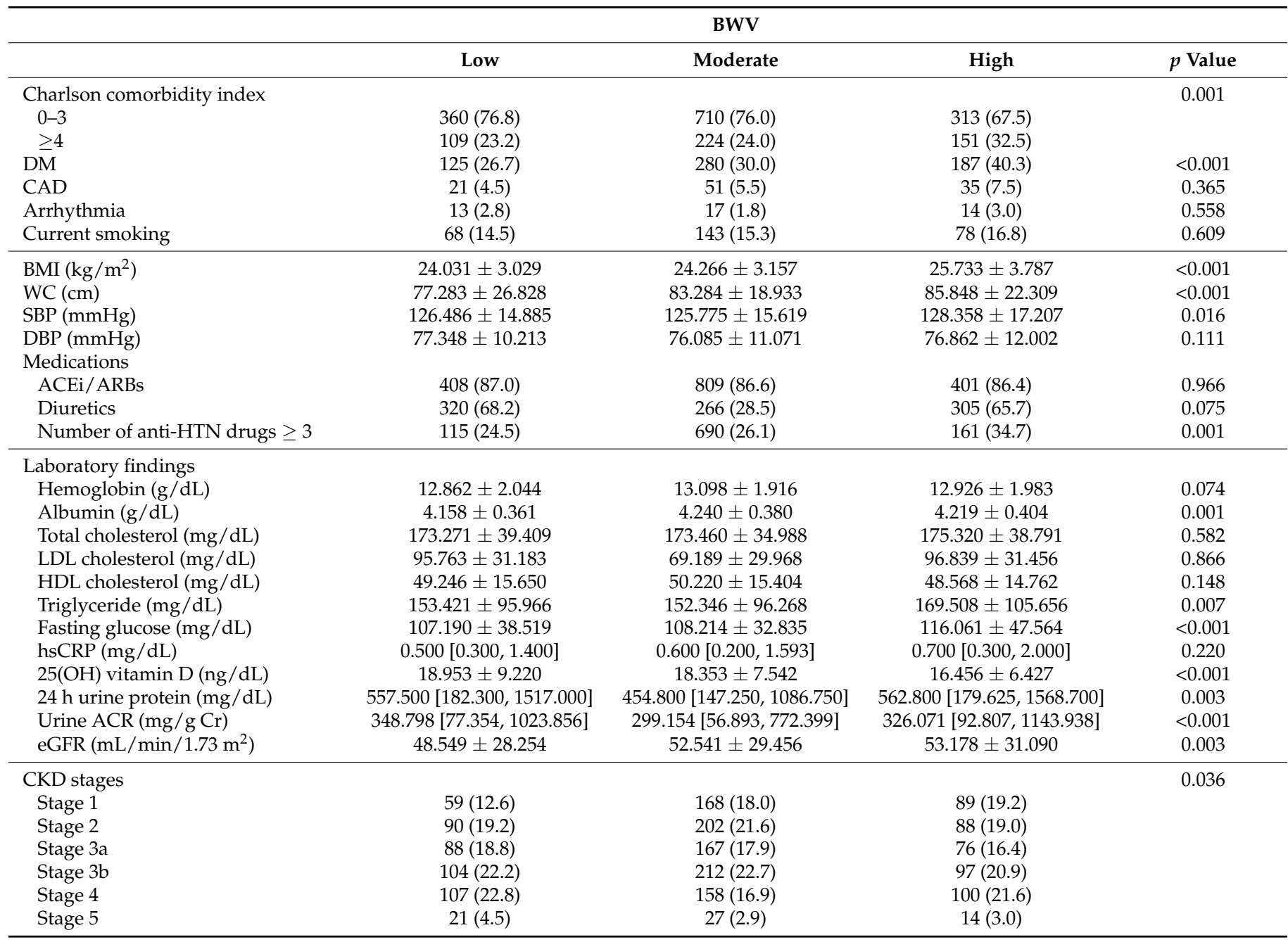

Values for categorical variables are given as number (percentage); values for continuous variables, as mean \pm standard deviation or median (interquartile range). Abbreviations: $\mathrm{ACEi}$, angiotensin-converting enzyme inhibitor; $\mathrm{ACR}$, albumin-to-creatinine ratio; $\mathrm{ARB}$, angiotensin receptor blocker; $\mathrm{BMI}$, body mass index; $\mathrm{CAD}$, coronary artery disease; CCI, Charlson comorbidity index; CKD, chronic kidney disease; DBP, diastolic blood pressure; DM, diabetes mellitus; eGFR, estimated glomerular filtration rate; hsCRP, high-sensitivity C-reactive protein; SBP, systolic blood pressure; WC, waist circumference.

\subsection{High BWV Is Associated with Adverse CV Outcomes in Patients with Pre-Dialysis CKD}

To address the association of BWV and the study outcomes, Kaplan-Meier survival was analyzed by BWV (Figure 2). The survival curves for the composite outcome (Log rank, $p=0.115$ ) and fatal and non-fatal CV events (Log rank, $p=0.126)$ revealed no significant differences among the groups by $\mathrm{BWV}$, whereas the all-cause death-free survival significantly differs among the groups (Log rank, $p=0.023$ ). To determine whether BWV is independently associated with CV outcomes in patients with pre-dialysis CKD, Cox proportional hazard regression models were analyzed. (Table 2). Compared to the subjects with moderated BWV, those with high BWV were associated with increased risks of the composite outcome (adjusted HR 1.738,95\% CI 1.065 to 2.847, $p=0.027$ ), fatal and non-fatal CV events (adjusted HR 1.845, 95\% CI 1.136 to $2.996, p=0.013$ ), and all-cause mortality (adjusted HR 1.861, 95\% CI 1.101 to 3.145, $p=0.020$ ). In the subgroup analyses (Table 3), high BWV was associated with the composite outcome in the subjects with age $\geq 60$ years (adjusted HR 2.361, 95\% CI 1.139 to $4.897, p=0.021$ ), medical history of DM (adjusted HR $2.199,95 \%$ CI 1.091 to $4.431, p=0.028$ ), and total number of antihypertensive drugs $\geq 3$ (adjusted HR 6.172, 95\% CI 1.394 to 27.333, $p=0.017$ ). Interestingly, both low (adjusted HR 2.542, 95\% CI 1.072 to $6.024, p=0.034$ ) and high (adjusted HR 2.545, 95\% CI 1.181 to 
$5.486, p=0.017) \mathrm{BWV}$ were associated with the composite outcomes in those without use of diuretics. Low BWV was also associated with the composite outcomes in those with UACR $<300 \mathrm{mg} / \mathrm{g}$ (adjusted HR 3.509, 95\% CI 1.028 to 9.105, $p=0.045$ ). The association of high BWV with fatal and non-fatal CV events (Supplemental Table S5) were also significant in the subjects with age $\geq 60$ years (adjusted HR 2.191, 95\% CI 1.054 to 4.557, $p=0.036$ ), male gender (adjusted HR 1.865, 95\% CI 1.020 to $3.412, p=0.043$ ), medical history of DM (adjusted HR 2.300, 95\% CI 1.147 to $4.612, p=0.019$ ), BMI $\geq 25 \mathrm{~kg} / \mathrm{m}^{2}$ (adjusted HR 3.048, $95 \%$ CI 1.181 to $7.869, p=0.021$ ), total number of antihypertensive drugs $\geq 3$ (adjusted HR 7.993, 95\% CI 2.145 to $29.779, p=0.002$ ), and eGFR $<45 \mathrm{~mL} / \mathrm{min} . / 1.73 \mathrm{~m}^{2}$ (adjusted HR 2.195, 95\% CI 1.051 to $4.582, p=0.036$ ). Only high BWV were associated with the fatal and non-fatal CV events in those without use of diuretics (adjusted HR 2.515, 95\% CI 1.165 to $5.428, p=0.019$ ). In the subgroup analyses for all-cause mortality (Supplemental Table S6), high BWV was associated with increased risk of all-cause mortality only in the subjects with age $\geq 60$ years (adjusted HR $2.438,95 \%$ CI 1.324 to $4.499, p=0.004$ ). Taken together, high BWV is significantly associated with adverse $\mathrm{CV}$ outcomes in patients with pre-dialysis CKD.
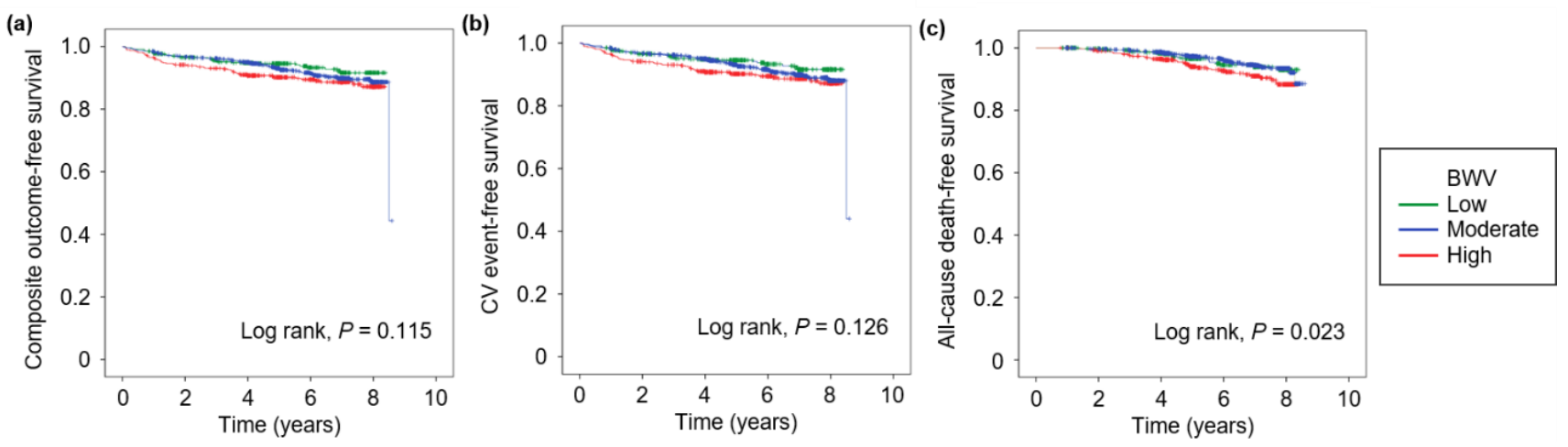

Figure 2. Kaplan-Meier survival curve for the outcomes by BWV. The probability of composite outcome- (a), fatal and non-fatal CV event- (b), and all-cause death- (c) free survivals by BW variability. $p$ values by Log rank test. Abbreviations: BWV, body weight variability.

Table 2. Cox proportional hazards regression of BWV for the outcomes.

\begin{tabular}{|c|c|c|c|c|c|}
\hline & & \multicolumn{2}{|c|}{ Unadjusted } & \multicolumn{2}{|l|}{ Adjusted } \\
\hline & & HR (95\% CIs) & $p$ Value & HR (95\% CIs) & $p$ Value \\
\hline \multirow{3}{*}{$\begin{array}{l}\text { Composite } \\
\text { outcome }\end{array}$} & Low BWV & $1.236(0.743,2.057)$ & 0.414 & $1.444(0.790,2.642)$ & 0.233 \\
\hline & Moderate BWV & Reference & & Reference & \\
\hline & High BWV & $1.789(1.195,2.679)$ & 0.005 & $1.738(1.065,2.847)$ & 0.027 \\
\hline \multirow{3}{*}{$\begin{array}{l}\text { Fatal and non-fatal } \\
\text { CV events }\end{array}$} & Low BWV & $1.283(0.771,2.133)$ & 0.337 & $1.593(0.882,2.884)$ & 0.123 \\
\hline & Moderate BWV & Reference & & Reference & \\
\hline & High BWV & $1.827(1.221,2.735)$ & 0.003 & $1.845(1.136,2.996)$ & 0.013 \\
\hline \multirow{3}{*}{ All-cause mortality } & Low BWV & $1.008(0.543,1.870)$ & 0.980 & $0.930(0.493,1.753)$ & 0.822 \\
\hline & Moderate BWV & Reference & & Reference & \\
\hline & High BWV & $1.702(1.043,2.776)$ & 0.033 & $1.861(1.101,3.145)$ & 0.020 \\
\hline
\end{tabular}

Models were adjusted for age, gender, Charlson comorbidity index, history of DM, smoking history, BMI, SBP, DBP, Medications (ACEi/ARBs, diuretics, number of antihypertensive drugs), hemoglobin, albumin, HDL-cholesterol, triglycerides, fasting serum glucose, hs-CRP, 25(OH) vitamin D levels, eGFR, and $24 \mathrm{~h}$ urine protein. Abbreviations: BWV, body weight variability; CV, cardiovascular; CI, confidence interval. 
Table 3. Cox proportional hazards regression of BWV for the composite outcome in various subgroups.

\begin{tabular}{|c|c|c|c|c|c|}
\hline & & \multicolumn{2}{|c|}{ Unadjusted } & \multicolumn{2}{|c|}{ Adjusted } \\
\hline & & HR (95\% CIs) & $p$ Value & HR (95\% CIs) & $p$ Value \\
\hline \multirow{3}{*}{ Age $<60$ years } & Low BWV & $1.811(0.814,4.030)$ & 0.146 & $0.595(0.183,1.938)$ & 0.204 \\
\hline & Moderate BWV & Reference & & Reference & \\
\hline & High BWV & $1.675(0.879,3.195)$ & 0.117 & $0.782(0.265,2.302)$ & 0.782 \\
\hline \multirow{3}{*}{ Age $\geq 60$ years } & Low BWV & $1.057(0.541,2.065)$ & 0.870 & $1.048(0.429,2.559)$ & 0.919 \\
\hline & Moderate BWV & Reference & & Reference & \\
\hline & High BWV & $2.089(1.234,3.539)$ & 0.006 & $2.361(1.139,4.897)$ & 0.021 \\
\hline \multirow{3}{*}{ Male } & Low BWV & $1.853(0.966,3.554)$ & 0.064 & $1.512(0.6323,3.614)$ & 0.353 \\
\hline & Moderate BWV & Reference & & Reference & \\
\hline & High BWV & $1.508(0.931,2.440)$ & 0.095 & $1.723(0.926,3.204)$ & 0.086 \\
\hline \multirow[t]{3}{*}{ Female } & Low BWV & $0.720(0.302,1.719)$ & 0.460 & $0.275(0.052,1.438)$ & 0.126 \\
\hline & Moderate BWV & Reference & & Reference & \\
\hline & High BWV & $2.606(1.187,5.720)$ & 0.017 & $0.271(0.049,1.490)$ & 0.133 \\
\hline \multirow[t]{3}{*}{$\mathrm{CCI} \leq 3$} & Low BWV & $0.883(0.395,1.978)$ & 0.763 & $0.879(0.287,2.695)$ & 0.821 \\
\hline & Moderate BWV & Reference & & Reference & \\
\hline & High BWV & $2.242(1.231,4.083)$ & 0.008 & $1.910(0.812,4.496)$ & 0.138 \\
\hline \multirow[t]{3}{*}{$\mathrm{CCI} \geq 4$} & Low BWV & $1.781(0.896,3.542)$ & 0.100 & $3.855(1.373,10.823)$ & 0.010 \\
\hline & Moderate BWV & Reference & & Reference & \\
\hline & High BWV & $1.499(0.841,2.670)$ & 0.169 & $1.364(0.589,3.157)$ & 0.469 \\
\hline \multirow[t]{3}{*}{$\mathrm{DM}(-)$} & Low BWV & $1.164(0.539,2.513)$ & 0.700 & $2.012(0.659,6.143)$ & 0.220 \\
\hline & Moderate BWV & Reference & & Reference & \\
\hline & High BWV & $1.952(1.002,3.800)$ & 0.049 & $1.429(0.531,3.842)$ & 0.480 \\
\hline \multirow[t]{3}{*}{$\mathrm{DM}(+)$} & Low BWV & $1.304(0.654,2.600)$ & 0.451 & $2.169(0.762,6.174)$ & 0.147 \\
\hline & Moderate BWV & Reference & & Reference & \\
\hline & High BWV & $1.620(0.947,2.769)$ & 0.078 & $2.199(1.091,4.431)$ & 0.028 \\
\hline \multirow[t]{3}{*}{$\mathrm{BMI}<25\left(\mathrm{~kg} / \mathrm{m}^{2}\right)$} & Low BWV & $1.452(0.785,2.687)$ & 0.235 & $2.175(0.953,4.965)$ & 0.065 \\
\hline & Moderate BWV & Reference & & Reference & \\
\hline & High BWV & $1.891(1.072,3.335)$ & 0.028 & $1.743(0.813,3.739)$ & 0.153 \\
\hline \multirow[t]{3}{*}{$\mathrm{BMI} \geq 25\left(\mathrm{~kg} / \mathrm{m}^{2}\right)$} & Low BWV & $0.898(0.341,2.367)$ & 0.829 & $1.203(0.283,5.125)$ & 0.802 \\
\hline & Moderate BWV & Reference & & Reference & \\
\hline & High BWV & $1.578(0.863,2.884)$ & 0.138 & $2.666(0.938,7.579)$ & 0.066 \\
\hline \multirow[t]{3}{*}{ Diuretics (-) } & Low BWV & $1.789(0.928,3.447)$ & 0.082 & $2.542(1.072,6.024)$ & 0.034 \\
\hline & Moderate BWV & Reference & & Reference & \\
\hline & High BWV & $3.037(1.659,5.558)$ & $<0.001$ & $2.545(1.181,5.486)$ & 0.017 \\
\hline \multirow[t]{3}{*}{ Diuretics (+) } & Low BWV & $0.824(0.353,1.921)$ & 0.653 & $0.531(0.148,1.914)$ & 0.334 \\
\hline & Moderate BWV & Reference & & Reference & \\
\hline & High BWV & $1.283(0.699,2.355)$ & 0.422 & $0.956(0.398,2.296)$ & 0.919 \\
\hline \multirow{3}{*}{$\begin{array}{c}\text { Number of } \\
\text { anti-HTN drugs } \leq 2\end{array}$} & Low BWV & $1.374(0.771,2.450)$ & 0.281 & $1.822(0.869,3.821)$ & 0.112 \\
\hline & Moderate BWV & Reference & & Reference & \\
\hline & High BWV & $1.641(0.983,2.738)$ & 0.058 & $1.670(0.838,3.328)$ & 0.145 \\
\hline \multirow{3}{*}{$\begin{array}{c}\text { Number of } \\
\text { anti-HTN drugs } \geq 3\end{array}$} & Low BWV & $0.640(0.187,2.187)$ & 0.477 & $1.451(0.159,13.227)$ & 0.741 \\
\hline & Moderate BWV & Reference & & Reference & \\
\hline & High BWV & $2.374(1.199,4.704)$ & 0.013 & $6.172(1.394,27.333)$ & 0.017 \\
\hline \multirow{3}{*}{$\begin{array}{c}\mathrm{eGFR} \geq 45 \\
\mathrm{~mL} / \mathrm{min} / 1.73 \mathrm{~m}^{2}\end{array}$} & Low BWV & $1.641(0.624,4.314)$ & 0.315 & $0.282(0.060,1.326)$ & 0.109 \\
\hline & Moderate BWV & Reference & & Reference & \\
\hline & High BWV & $1.956(1.049,3.649)$ & 0.035 & $2.066(0.855,4.991)$ & 0.107 \\
\hline \multirow{3}{*}{$\begin{array}{c}\mathrm{eGFR}<45 \\
\mathrm{~mL} / \mathrm{min} / 1.73 \mathrm{~m}^{2}\end{array}$} & Low BWV & $1.130(0.609,2.097)$ & 0.698 & $1.679(0.754,3.741)$ & 0.205 \\
\hline & Moderate BWV & Reference & & Reference & \\
\hline & High BWV & $1.822(1.058,3.318)$ & 0.031 & $1.626(0.775,3.413)$ & 0.199 \\
\hline
\end{tabular}


Table 3. Cont.

\begin{tabular}{cccccc}
\hline & & \multicolumn{2}{c}{ Unadjusted } & \multicolumn{2}{c}{ Adjusted } \\
\cline { 3 - 6 } & & HR (95\% CIs) & $p$ Value & HR (95\% CIs) & $p$ Value \\
\hline $\begin{array}{c}\text { Random urine ACR } \\
<300 \mathrm{mg} / \mathrm{g}\end{array}$ & $\begin{array}{c}\text { Low BWV } \\
\text { Moderate BWV } \\
\text { High BWV }\end{array}$ & $\begin{array}{c}1.603(0.734,3.501) \\
\text { Reference }\end{array}$ & 0.237 & $\begin{array}{c}3.059(1.028,9.105) \\
\text { Reference }\end{array}$ & 0.045 \\
& $2.150(1.154,4.006)$ & 0.016 & $2.308(0.977,5.454)$ & 0.057 \\
$\begin{array}{c}\text { Random urine ACR } \\
\geq 300 \mathrm{mg} / \mathrm{g}\end{array}$ & $\begin{array}{c}\text { Low BWV } \\
\text { Moderate BWV }\end{array}$ & $\begin{array}{c}0.967(0.485,1.926) \\
\text { Reference }\end{array}$ & 0.924 & $\begin{array}{c}0.964(0.380,2.447) \\
\text { Reference }\end{array}$ & 0.939 \\
& High BWV & $1.464(0.833,2.570)$ & 0.185 & $1.554(0.690,3.500)$ & 0.287 \\
\hline
\end{tabular}

Models were adjusted for age, gender, Charlson comorbidity index, history of DM, smoking history, BMI, SBP, DBP, Medications (ACEi/ARBs, diuretics, number of antihypertensive drugs), hemoglobin, albumin, HDL-cholesterol, triglycerides, fasting serum glucose, hs-CRP, 25(OH) vitamin D levels, eGFR, and $24 \mathrm{~h}$ urine protein. Abbreviations: ACR, Albumin-to-creatinine ratio; BMI, body mass index; CCI, Charlson comorbidity index; CI, confidence interval; DM, diabetes mellitus; HTN, hypertension; eGFR, estimated glomerular filtration rate.

\begin{abstract}
3.3. High BWV Is Associated with Adverse CV Outcomes Even in Patients without BW Gain or Loss during Follow-up Periods

To determine the association between BWV and the rate of longitudinal BW change, multinomial logistic regression models were analyzed (Supplemental Table S7), which demonstrated a robust correlation of high BWV with both BW decrease (adjusted OR 2.310, $95 \%$ CI 1.661 to $3.213, p<0.001$ ) and BW increase (adjusted OR 2.642, 95\% CI 1.912 to 3.652, $p<0.001)$. Inversely, low BWV was associated with reduced risks of either BW decrease (adjusted OR $0.429,95 \%$ CI 0.300 to $0.614, p<0.001$ ) or BW increase (adjusted OR 0.541, $95 \%$ CI 0.384 to $0.763, p<0.001)$. However, the scatter plot for the correlation of the rate of longitudinal BW change and BWV (Supplemental Figure S1) visualized that a substantial portion of the subjects experience only a limited range of longitudinal BW change, but concurrently show visit-to-visit BWV (i.e., scatter plots condensed in the midline with a distribution along the $\mathrm{Y}$-axis direction). Therefore, we decided to unveil the association of high BWV with adverse CV outcomes in the subjects without BW gain or loss (i.e., the subjects with BW maintenance) during follow-up periods ( $n=930)$. The analyses of Cox proportional hazard regression models (Table 4) demonstrated that high BWV is associated with increased risk of fatal and non-fatal CV events in patients with BW maintenance during follow-up periods (adjusted HR 2.755, 95\% CI 1.114 to $6.813, p=0.028$ ), suggesting that fluctuations in BW impose an independent risk of CV events even in the absence of longitudinal BW change.
\end{abstract}

Table 4. Cox proportional hazards regression of BWV for the outcomes in subjects with BW maintenance during followup periods.

\begin{tabular}{|c|c|c|c|c|c|}
\hline & & \multicolumn{2}{|c|}{ Unadjusted } & \multicolumn{2}{|l|}{ Adjusted } \\
\hline & & HR (95\% CIs) & $p$ Value & HR (95\% CIs) & $p$ Value \\
\hline \multirow{3}{*}{$\begin{array}{l}\text { Composite } \\
\text { outcome }\end{array}$} & Low BWV & $1.580(0.733,3.406)$ & 0.243 & $1.878(0.701,5.029)$ & 0.210 \\
\hline & Moderate BWV & Reference & & Reference & \\
\hline & High BWV & $1.754(0.872,3.528)$ & 0.115 & $2.239(0.816,6.148)$ & 0.118 \\
\hline \multirow{3}{*}{$\begin{array}{l}\text { Fatal and non-fatal } \\
\text { CV events }\end{array}$} & Low BWV & $1.762(0.846,3.669)$ & 0.130 & $2.430(0.958,6.166)$ & 0.062 \\
\hline & Moderate BWV & Reference & & Reference & \\
\hline & High BWV & $1.838(0.913,3.698)$ & 0.088 & $2.755(1.114,6.813)$ & 0.028 \\
\hline \multirow{3}{*}{ All-cause mortality } & Low BWV & $1.729(0.716,4.179)$ & 0.224 & $1.530(0.580,4.037)$ & 0.390 \\
\hline & Moderate BWV & Reference & & Reference & \\
\hline & High BWV & $1.377(0.438,4.324)$ & 0.584 & $1.111(0.309,3.999)$ & 0.871 \\
\hline
\end{tabular}

Models were adjusted for age, gender, Charlson comorbidity index, history of DM, smoking history, BMI, SBP, DBP, Medications (ACEi/ARBs, diuretics, number of antihypertensive drugs), hemoglobin, albumin, HDL-cholesterol, triglycerides, fasting serum glucose, hs-CRP, 25(OH) vitamin D levels, eGFR, and $24 \mathrm{~h}$ urine protein. Abbreviations: CI, confidence interval. 
To figure out the impact of the baseline BMI in the subjects with high BWV, the subjects with high BWV were further divided into those with BMI 20 to $24.9 \mathrm{~kg} / \mathrm{m}^{2}$ (i.e., near normal BMI) vs. those with BMI $<20 \mathrm{~kg} / \mathrm{m}^{2}$ or $\geq \mathrm{r} /$ mesds $^{2}$ (i.e., relatively underweight or obese) (Supplemental Table S8). Intriguingly, only the subjects with high BWV and $\mathrm{BMI}<20 \mathrm{~kg} / \mathrm{m}^{2}$ or $\geq \mathrm{r} / \mathrm{mectsn}^{2}$ were associated with increased risk of the composite outcome (adjusted HR 2.149, 95\% CI 1.107 to $4.173, p=0.024$ ), fatal and non-fatal CV events (adjusted HR 2.286, 95\% CI 1.180 to $4.426, p=0.014$ ), and all-cause mortality (adjusted HR $2.124,95 \%$ CI 1.101 to $4.095, p=0.025$ ), indicating a significant impact of the baseline BMI in addition to BWV on the prognosis of the patients with pre-dialysis CKD.

\section{Discussion}

In the present study, we discovered that high BWV is significantly associated with adverse $C V$ outcomes in patients with pre-dialysis CKD. We also demonstrated that BWV is associated with longitudinal of BW gain or loss in patients with CKD. Importantly, we proved that high BWV is associated with adverse CV outcomes, even in patients without significant BW gain or loss during follow-up periods.

In the current study, we found that BWV is associated with both longitudinal BW gain and loss in patients with CKD, rather than a unidirectional association toward BW gain or BW loss. This suggests the multifaceted nature of the progression in the body composition during the course of CKD. Indeed, a recent cohort study reported that both BW gain and loss are associated with adverse outcomes in patients with pre-dialysis CKD [19]. Meanwhile, in the present study, we primarily highlighted on the visit-to-visit fluctuation in BW, rather than longitudinal trends in BW, provided that several events that promote BW gain or loss may differ among each follow-up visit, in which case the BWV should be high, even though the BW slope might be blunted. Therefore, a potential strength of BWV over longitudinal BW change may be a sensitive detection of vulnerable subjects who are at high risk of $C V$ events. In this context, it is of interest to note that high BWV is associated with adverse $\mathrm{CV}$ outcomes, even in patients with $\mathrm{BW}$ maintenance during follow-up periods, as this suggests a prognostic impact of BWV independent of longitudinal BW change.

It is intriguing that, although high BWV was robustly associated adverse outcomes, low BWV was also associated with high risk of the composite outcome in certain clinical contexts (e.g., urine ACR $<300 \mathrm{mg} / \mathrm{g}$ ). We speculate that this results from the complex nature of homeostasis in body composition during the course of CKD, which may justify $\mathrm{BWV}$ to some degree. Indeed, in the present study, the risks of adverse outcomes were almost consistently lowest in subjects with 'moderate', but not 'low', BWV. The precise mechanism of how a moderate, not low, degree of BWV predicts the best outcomes in patients with pre-dialysis CKD should be further elucidated.

The mechanism of how BWV is associated with adverse CV outcomes is another remaining question. One possible explanation is the association of BWV and coronary artery calcification (CAC), although the association has not been validated yet. Previous studies reported that central adiposity is strongly associated with CAC in general population [20] and in CKD patients [21,22]. Inversely, malnutrition-inflammation is associated with a higher CAC score in diabetic CKD patients [23], as well as in patients with chronic dialysis $[24,25]$. As BWV is a sensitive read-out of conditions associated with BW gain or loss in CKD population, we speculate that BWV may predict the risk of CAC, delineating its association with adverse CV outcomes. Another explanation is the association of BWV and heart failure (HF). It has been reported that the fluctuations in BW [26] or anthropometric indices [27], such as BMI and the waist-to-hip ratio, and BW and is associated with increased mortality in patients with HF. As the renal function of the subjects in those studies is relatively reserved, the association of BWV and HF should be further evaluated.

There are a number of limitations in this study. First, we are not able to clarify the causal relationship between high BWV and adverse CV outcomes, because of the observational nature of the current study. Second, despite the clear impact of high BWV on the adverse $\mathrm{CV}$ outcomes, the precise mechanism should be further addressed. Third, as 
this cohort study enrolled only ethnic Koreans, a precaution is required to extrapolate the data in the present study to other populations. Fourth, there is a potential risk of multiple testing burden in the current study, as the multiple outcomes have been analyzed.

In conclusion, we report that high BWV is significantly associated with adverse CV outcomes in patients with pre-dialysis CKD. Our results suggest that BWV is associated with longitudinal of BW gain or loss in patients with CKD, while high BWV is associated with adverse $\mathrm{CV}$ outcomes, even in patients without BW gain or loss during followup periods.

Supplementary Materials: The following are available online at https:/ /www.mdpi.com/article/10 $.3390 /$ nu13103381/s1, Table S1: Baseline characteristics of study participants by BW slope. Table S2: Raw numbers for the composite outcome by enrollment sites. Table S3: Raw numbers for the composite outcome by subgroups. Table S4: Charlson Comorbidity Index components of study participants by BWV. Table S5: Cox proportional hazards regression of BWV for the fatal and non-fatal $\mathrm{CV}$ events in various subgroups. Table S6: Cox proportional hazards regression of BWV for all-cause mortality in various subgroups. Table S7: Multinomial logistic regression of BWV for longitudinal BW change. Table S8: Cox proportional hazards regression of BMI in addition to BWV for the outcomes. Figure S1: Scatter plot for the correlations of BW slope with BWV.

Author Contributions: Conceptualization, S.H.S.; methodology, S.H.S., T.R.O., H.S.C. and C.S.K.; formal analysis, S.H.S.; resources, E.H.B., S.K.P., Y.-S.K., Y.H.K., K.H.C. and K.-H.O.; data curation, S.H.S.; writing—original draft preparation, S.H.S.; writing—review and editing, S.H.S., S.K.M. and S.W.K.; supervision, S.K.M. and S.W.K.; funding acquisition, C.S.K., K.-H.O. and S.W.K. All authors have read and agreed to the published version of the manuscript.

Funding: This work was supported by the Research Program funded by the Korea Centers for Disease Control and Prevention (2011E3300300, 2012E3301100, 2013E3301600, 2013E3301601, 2013E3301602, 2016E3300200, 2016E3300201, 2016E3300202, and 2019E320100), by a grant of the Korea Health Technology R\&D Project through the Korea Health Industry Development Institute (KHIDI), funded by the Ministry of Health \& Welfare, Republic of Korea (HR20C0021), and a grant (BCRI20062) of Chonnam National University Hospital Biomedical Research Institute.

Institutional Review Board Statement: The study protocol was approved by the Institutional Review Board at each participating clinical center [Seoul National University Hospital (1104-089-359), Seoul National University Bundang Hospital (B-1106/129-008), Yonsei University Severance Hospital (4-2011-0163), Kangbuk Samsung Medical Center (2011-01-076), Seoul St. Mary's Hospital (KC11OIMI0441), Gil Hospital (GIRBA2553), Eulji General Hospital (201105-01), Chonnam National University Hospital (CNUH-2011-092), and Busan Paik Hospital (11-091)].

Informed Consent Statement: Informed consent was obtained from all subjects involved in the study.

Data Availability Statement: Not applicable.

Acknowledgments: KNOW-CKD: Study Group Clinical Centers. Seoul National University, Curie Ahn, Kook-Hwan Oh, Dong Wan Chae, Ho Jun Chin, Hayne Cho Park, Seungmi Lee, Hyun Hwa Jang, and Hyun Jin Cho. Yonsei University, Severance Hospital, Kyu Hun Choi, Seung Hyeok Han, Tae Hyun Yoo, and Mi Hyun Yu. Kangbuk Samsung Medical Center, Kyubeck Lee, and Sooyeon Jin. The Catholic University of Korea, Seoul St. Mary's Hospital, Yong-Soo Kim, and Sol Ji Kim. Gachon University, Gil Hospital, Wookyung Chung, Youkyoung Jang, and Ji Hye Park. Eulji University, Eulji General Hospital. Young-Hwan Hwang, Su-Ah Sung, and Jeong Ok So. Chonnam University, Soo Wan Kim, and Ji Seon Lee. Inje University, Pusan Paik Hospital, Yeong Hoon Kim, Sun Woo Kang, and Yun Jin Kim. Epidemiology and Biostatistics. Department of Preventive Medicine, Seoul National University College of Medicine, Byung-Joo Park, Sue Kyung Park, and Juyeon Lee. Coordinating Center. Medical Research Collab-orating Center, Seoul National University Hospital and Seoul National University College of Medicine, Joongyub Lee, Dayeon Nam, Soohee Kang, MSc and Heejung Ahn. Cen-tral Laboratory, Donghee Seo, Lab Genomics, Korea and Dae Yeon Cho, Lab Genomics, Korea. Biobank. Korea Biobank, Korea Centers for Disease Control and Prevention, Osong, Korea. Korea Center for Disease Control and Prevention, Dukhyoung Lee, Hyekyung Park, (Project Officer), Eunkyeong Jung (Project Officer), Suyeon Jeong, Eunmi Ahn and Sil-Hea Sung.

Conflicts of Interest: The authors declare no conflict of interest. 


\section{References}

1. Zha, Y.; Qian, Q. Protein Nutrition and Malnutrition in CKD and ESRD. Nutrients 2017, 9, 208. [CrossRef] [PubMed]

2. Lee, S.W.; Kim, Y.S.; Kim, Y.H.; Chung, W.; Park, S.K.; Choi, K.H.; Ahn, C.; Oh, K.H. Dietary Protein Intake, Protein Energy Wasting, and the Progression of Chronic Kidney Disease: Analysis from the KNOW-CKD Study. Nutrients 2019, 11, 121. [CrossRef] [PubMed]

3. Jagadeswaran, D.; Indhumathi, E.; Hemamalini, A.J.; Sivakumar, V.; Soundararajan, P.; Jayakumar, M. Inflammation and nutritional status assessment by malnutrition inflammation score and its outcome in pre-dialysis chronic kidney disease patients Clin. Nutr. 2019, 38, 341-347. [CrossRef]

4. Sung, C.C.; Liao, M.T.; Chao, C.T. Independent Determinants of Appetite Impairment among Patients with Stage 3 or Higher Chronic Kidney Disease: A Prospective Study. Nutrients 2021, 13, 2863. [CrossRef]

5. Park, S.; Lee, C.J.; Jhee, J.H.; Yun, H.R.; Kim, H.; Jung, S.Y.; Kee, Y.K.; Yoon, C.Y.; Park, J.T.; Kim, H.C.; et al. Extracellular Fluid Excess Is Significantly Associated With Coronary Artery Calcification in Patients with Chronic Kidney Disease. J. Am. Heart Assoc. 2018, 7, e008935. [CrossRef]

6. Kim, Y.J.; Jeon, H.J.; Kim, Y.H.; Jeon, J.; Ham, Y.R.; Chung, S.; Choi, D.E.; Na, K.R.; Lee, K.W. Overhydration measured by bioimpedance analysis and the survival of patients on maintenance hemodialysis: A single-center study. Kidney Res. Clin. Pract. 2015, 34, 212-218. [CrossRef] [PubMed]

7. Khan, Y.H.; Sarriff, A.; Adnan, A.S.; Khan, A.H.; Mallhi, T.H. Diuretics prescribing in chronic kidney disease patients: Physician assessment versus bioimpedence spectroscopy. Clin. Exp. Nephrol. 2017, 21, 488-496. [CrossRef]

8. Lissner, L.; Odell, P.M.; D'Agostino, R.B.; Stokes, J., 3rd; Kreger, B.E.; Belanger, A.J.; Brownell, K.D. Variability of body weight and health outcomes in the Framingham population. N. Engl. J. Med. 1991, 324, 1839-1844. [CrossRef]

9. Park, K.Y.; Hwang, H.S.; Cho, K.H.; Han, K.; Nam, G.E.; Kim, Y.H.; Kwon, Y.; Park, Y.G. Body Weight Fluctuation as a Risk Factor for Type 2 Diabetes: Results from a Nationwide Cohort Study. J. Clin. Med. 2019, 8, 950. [CrossRef]

10. Oh, T.J.; Moon, J.H.; Choi, S.H.; Lim, S.; Park, K.S.; Cho, N.H.; Jang, H.C. Body-Weight Fluctuation and Incident Diabetes Mellitus, Cardiovascular Disease, and Mortality: A 16-Year Prospective Cohort Study. J. Clin. Endocrinol. Metab. 2019, 104, 639-646. [CrossRef]

11. Lim, Y.M.; Yang, P.S.; Jang, E.; Yu, H.T.; Kim, T.H.; Uhm, J.S.; Kim, J.Y.; Pak, H.N.; Lee, M.H.; Joung, B.; et al. Body Mass Index Variability and Long-term Risk of New-Onset Atrial Fibrillation in the General Population: A Korean Nationwide Cohort Study. Mayo Clin. Proc. 2019, 94, 225-235. [CrossRef]

12. Nam, G.E.; Kim, W.; Han, K.; Lee, C.W.; Kwon, Y.; Han, B.; Park, S.; Park, J.H.; Kim, Y.H.; Kim, D.H.; et al. Body Weight Variability and the Risk of Cardiovascular Outcomes and Mortality in Patients with Type 2 Diabetes: A Nationwide Cohort Study. Diabetes Care 2020, 43, 2234-2241. [CrossRef]

13. Yeboah, P.; Hsu, F.C.; Bertoni, A.G.; Yeboah, J. Body Mass Index, Change in Weight, Body Weight Variability and Outcomes in Type 2 Diabetes Mellitus (from the ACCORD Trial). Am. J. Cardiol. 2019, 123, 576-581. [CrossRef] [PubMed]

14. Kim, M.N.; Han, K.; Yoo, J.; Ha, Y.; Chon, Y.E.; Lee, J.H.; Simon, T.G.; Chan, A.T.; Hwang, S.G. Body weight variability and the risk of cardiovascular outcomes in patients with nonalcoholic fatty liver disease. Sci. Rep. 2021, 11, 9154. [CrossRef] [PubMed]

15. Bangalore, S.; Fayyad, R.; Laskey, R.; DeMicco, D.A.; Messerli, F.H.; Waters, D.D. Body-Weight Fluctuations and Outcomes in Coronary Disease. N. Engl. J. Med. 2017, 376, 1332-1340. [CrossRef] [PubMed]

16. Bangalore, S.; Fayyad, R.; DeMicco, D.A.; Colhoun, H.M.; Waters, D.D. Body Weight Variability and Cardiovascular Outcomes in Patients With Type 2 Diabetes Mellitus. Circ. Cardiovasc. Qual. Outcomes 2018, 11, e004724. [CrossRef] [PubMed]

17. Oh, K.H.; Park, S.K.; Park, H.C.; Chin, H.J.; Chae, D.W.; Choi, K.H.; Han, S.H.; Yoo, T.H.; Lee, K.; Kim, Y.S.; et al. KNOW-CKD (KoreaN cohort study for Outcome in patients With Chronic Kidney Disease): Design and methods. BMC Nephrol. 2014, 15, 80. [CrossRef] [PubMed]

18. Levey, A.S.; Stevens, L.A.; Schmid, C.H.; Zhang, Y.L.; Castro, A.F., 3rd; Feldman, H.I.; Kusek, J.W.; Eggers, P.; Van Lente, F.; Greene, T.; et al. A new equation to estimate glomerular filtration rate. Ann. Intern. Med. 2009, 150, 604-612. [CrossRef]

19. Ryu, H.; Hong, Y.; Kang, E.; Kang, M.; Kim, J.; Oh, Y.K.; Yang, S.J.; Yang, Y.J.; Park, S.K.; Chung, W.; et al. Rapid Weight Change over Time Is a Risk Factor for Adverse Outcomes in Patients With Predialysis Chronic Kidney Disease: A Prospective Cohort Study. J. Ren. Nutr. 2021. [CrossRef] [PubMed]

20. Park, J.; Lee, E.S.; Lee, D.Y.; Kim, J.; Park, S.E.; Park, C.Y.; Lee, W.Y.; Oh, K.W.; Park, S.W.; Rhee, E.J. Waist Circumference as a Marker of Obesity Is More Predictive of Coronary Artery Calcification than Body Mass Index in Apparently Healthy Korean Adults: The Kangbuk Samsung Health Study. Endocrinol. Metab. 2016, 31, 559-566. [CrossRef]

21. Lee, M.J.; Park, J.T.; Park, K.S.; Kwon, Y.E.; Han, S.H.; Kang, S.W.; Choi, K.H.; Oh, K.H.; Park, S.K.; Chae, D.W.; et al. Normal body mass index with central obesity has increased risk of coronary artery calcification in Korean patients with chronic kidney disease. Kidney Int. 2016, 90, 1368-1376. [CrossRef] [PubMed]

22. Aoqui, C.; Cuppari, L.; Kamimura, M.A.; Canziani, M.E. Increased visceral adiposity is associated with coronary artery calcification in male patients with chronic kidney disease. Eur. J. Clin. Nutr. 2013, 67, 610-614. [CrossRef]

23. Cano-Megías, M.; Bouarich, H.; Guisado-Vasco, P.; Pérez Fernández, M.; de Arriba-de la Fuente, G.; Álvarez-Sanz, C.; RodríguezPuyol, D. Coronary artery calcification in patients with diabetes mellitus and advanced chronic kidney disease. Endocrinol. Diabetes Nutr. 2019, 66, 297-304. [CrossRef] 
24. Turkmen, K.; Kayikcioglu, H.; Ozbek, O.; Solak, Y.; Kayrak, M.; Samur, C.; Anil, M.; Zeki Tonbul, H. The relationship between epicardial adipose tissue and malnutrition, inflammation, atherosclerosis/calcification syndrome in ESRD patients. Clin. J. Am. Soc. Nephrol. 2011, 6, 1920-1925. [CrossRef] [PubMed]

25. Srivaths, P.R.; Silverstein, D.M.; Leung, J.; Krishnamurthy, R.; Goldstein, S.L. Malnutrition-inflammation-coronary calcification in pediatric patients receiving chronic hemodialysis. Hemodial. Int. 2010, 14, 263-269. [CrossRef] [PubMed]

26. Li, Y.; Yu, Y.; Wu, Y.; Liang, W.; Dong, B.; Xue, R.; Dong, Y.; Zhu, W.; Huang, P. Association of Body-Weight Fluctuation with Outcomes in Heart Failure With Preserved Ejection Fraction. Front. Cardiovasc. Med. 2021, 8, 689591. [CrossRef] [PubMed]

27. Aranda, R.M.; Puerto, J.L.; Andrey, J.L.; Escobar, M.A.; García-Egido, A.; Romero, S.P.; Pedrosa, M.J.; Gómez, F. Fluctuations of the anthropometric indices and mortality of patients with incident heart failure: A prospective study in the community. Int. J. Clin. Pract. 2015, 69, 169-179. [CrossRef] [PubMed] 